\title{
Post-synthetically modified porous covalent framework (PCF) for high proton conduction
}

Partha Samanta $^{1}$, Aamod Desai ${ }^{1}$, Bihag Anothumakkool ${ }^{2}$, Mandar Shirolkar ${ }^{3}$, Avishek Karmakar ${ }^{1}$, Sreekumar Kurungot ${ }^{2}$, Sujit Ghosh ${ }^{1}$ ${ }^{1}$ Department Of Chemistry, Indian Institute Of Science Education And Research (IIS, Pune, India, ${ }^{2}$ National Chemical Laboratory (NCL), Pune, India, ${ }^{3}$ University of Technology of China, Hefei, China

E-mail: partha.samanta@students.iiserpune.ac.in

An Ether linkage based highly chemically stable porous covalent framework (PCF-1) has been synthesized. Owing to the acid stability of PCF-1, post-synthetic covalent modification was executed with a simple room temperature reaction for the introduction of free pendant sulphonic acid (-SO3H) groups. Introduction of free Brønsted acid groups into the porous network of PCF-1 forms suitable network which performs as proton conducting pathway. The covalently modified compound (PCF-1-SO3H) presents a remarkably high proton conductivity (ca. $0.026 \mathrm{Scm}-1$ at $30{ }^{\circ} \mathrm{C}$ and $95 \% \mathrm{RH}$ ) which is comparable with commercially available Nafion-based material, with a 130 fold enhancement in proton conductivity over the parent compound. Notably, this value stands as the highest known value in the regime of post-synthetically modified porous organic frameworks. Activation energy of sulphonated PCF-1 has been calculated from temperature dependent impedance analysis at $95 \% \mathrm{RH}$ and it has been found to be $0.19 \mathrm{eV}$, which indicates Grotthuss mechanism is playing the role in proton conduction. Low activation energy for proton conducting materials also required for device fabrication and real time applications.

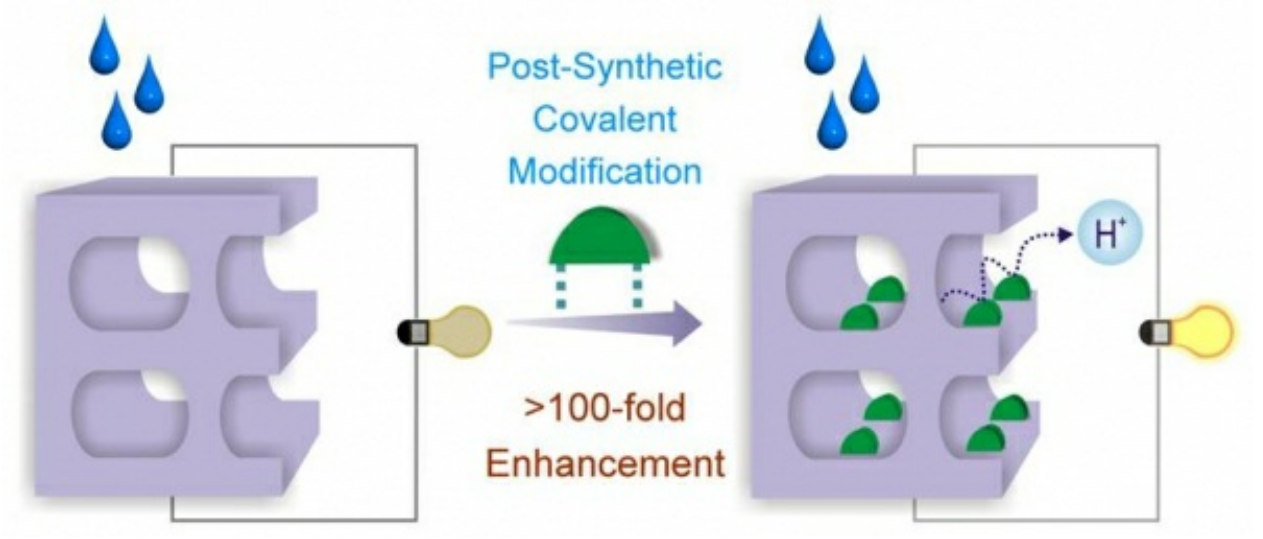

Keywords: Proton Conduction, Post-synthetic Modification, Porous Covalent Framework 